Submitted to the Astrophysical Journal

Preprint typeset using $\mathrm{LAT}_{\mathrm{E} X}$ style emulateapj v. 5/25/10

\title{
THE NEUTRON STAR MASS DISTRIBUTION
}

\author{
Bülent Kiziltan ${ }^{1,2}$, Athanasios Kottas ${ }^{3},{\text { Maria De } \text { Yoreo }^{3} \text { \& Stephen E. Thorseto }}^{2,4}$ \\ Submitted to the Astrophysical Journal
}

\begin{abstract}
In recent years, the number of pulsars with secure mass measurements has increased to a level that allows us to probe the underlying neutron star (NS) mass distribution in detail. We critically review the radio pulsar mass measurements. For the first time, we are able to analyze a sizable population of NSs with a flexible modeling approach that can effectively accommodate a skewed underlying distribution and asymmetric measurement errors. We find that NSs that have evolved through different evolutionary paths reflect distinctive signatures through dissimilar distribution peak and mass cutoff values. NSs in double neutron star and neutron star-white dwarf systems show consistent respective peaks at $1.33 \mathrm{M}_{\odot}$ and $1.55 \mathrm{M}_{\odot}$ suggesting significant mass accretion $\left(\Delta m \approx 0.22 \mathrm{M}_{\odot}\right)$ has occurred during the spin-up phase. The width of the mass distribution implied by double NS systems is indicative of a tight initial mass function while the inferred mass range is significantly wider for NSs that have gone through recycling. We find a mass cutoff at $\sim 2.1 \mathrm{M}_{\odot}$ for $\mathrm{NSs}$ with white dwarf companions which establishes a firm lower bound for the maximum NS mass. This rules out the majority of strange quark and soft equation of state models as viable configurations for NS matter. The lack of truncation close to the maximum mass cutoff along with the skewed nature of the inferred mass distribution both enforce the suggestion that the $2.1 \mathrm{M}_{\odot}$ limit is set by evolutionary constraints rather than nuclear physics or general relativity, and the existence of rare super-massive NSs is possible. Subject headings: binaries: general — methods: statistical — pulsars: general — stars: fundamental parameters — stars: neutron — stars: statistics
\end{abstract}

\section{INTRODUCTION}

The mass of a neutron star (NS) has been a prime focus of compact objects astrophysics since the discovery of neutrons. Soon after Chadwick's Letter on the "Possible existence of a neutron" (1932), heated discussions around the world started to take place on the potential implications of the discovery. In 1932, during one of these discussions in Copenhagen, Landau shared his views with Rosenfeld and Bohr where he anticipated the existence of a dense-compact star composed primarily of neutrons (e.g., Shapiro \& Teukolsky 1983, p. 242). The prediction was not officially announced until Baade \& Zwicky published their work where the phrase "neutron star" appeared in the literature for the first time (Baade \& Zwicky 1934a). Their following work explained the possible evolutionary process leading to the production of a NS and the physics that simultaneously constrains the mass and radius in more detail (Baade \& Zwicky 1934b,c).

The ensuing discussions were primarily focused on the mass of these dense objects. In 1931, Chandrasekhar had already published his original work in which he calculates the upper mass limit of an "ideal" white dwarf as $0.91 \mathrm{M}_{\odot}$, while the following year, Landau intuitively predicted that a limiting mass should exist close to $1.5 \mathrm{M}_{\odot}$ (Landau 1932). Following the works

\footnotetext{
${ }^{1}$ Harvard-Smithsonian Center for Astrophysics, 60 Garden Street, Cambridge, MA 02138; e-mail: bkiziltan@cfa.harvard.edu

${ }^{2}$ Department of Astronomy \& Astrophysics, University of California \& UCO/Lick Observatory, Santa Cruz, CA 95064

${ }^{3}$ Department of Applied Mathematics and Statistics, University of California, Santa Cruz, CA 95064

4 Department of Physics, Willamette University, Salem, OR 97031
}

of Chandrasekhar and Landau, and using the formalism developed by Tolman, Oppenheimer \& Volkoff predicted an upper mass limit for NSs to be $0.7-3.4 \mathrm{M}_{\odot}$ (Tolman 1939; Oppenheimer \& Volkoff 1939).

Since then, continuing discussions on the mass range a NS can attain have spawned a vast literature (e.g., Rhoades \& Ruffini 1974; Joss \& Rappaport 1976; Thorsett \& Chakrabarty 1999; Baumgarte et al. 2000; Schwab et al. 2010, and references therein).

Masses of NSs at birth are tuned by the intricate details of the astrophysical processes that drive core collapse and supernova explosions (Timmes et al. 1996). The birth mass is therefore of particular interest to those who study these nuclear processes. An earlier attempt by Finn (1994) finds that NSs should predominantly fall in the 1.3-1.6 $\mathrm{M}_{\odot}$ mass range. The most comprehensive work to date by Thorsett \& Chakrabarty (1999) finds that the mass distribution of observed pulsars are consistent with $M=1.38_{+0.10}^{-0.06} \mathrm{M}_{\odot}$, a remarkably narrow mass range. The recent work of Schwab et al. (2010) on the other hand, argues that there is evidence for multi-modality in the NS birth mass distribution (for discussion, see $\S 6$ ).

The maximum possible mass of a NS has attracted particular attention because it delineates the low mass limit of stellar mass black holes (Rhoades \& Ruffini 1974; Fryer \& Kalogera 2001). When combined with measurements of NS radii, it also provides a distinctive insight into the structure of matter at supranuclear densities (Cook et al. 1994; Haensel 2003; Lattimer \& Prakash 2004, 2007). Although more modern values theoretically predict a maximum NS mass of $M_{\max } \approx$ 2.2-2.9 $\mathrm{M}_{\odot} \quad$ (Bombaci 1996; Kalogera \& Baym 1996; Heiselberg \& Pandharipande 2000), it is still unclear whether very stiff equations of states (EOSs) that stably 
sustain cores up to the general relativity limit $\left(\sim 3 \mathrm{M}_{\odot}\right)$ can exist.

Recent observations of pulsars in the Galactic plane as well as globular clusters suggest that there may be, in fact, NSs with masses significantly higher than the canonical value of $1.4 \mathrm{M}_{\odot}$ (e.g., Champion et al. 2008; Ransom et al. 2005; Freire 2008; Freire et al. 2008a,b). NSs in X-ray binaries also show systematic deviations from the canonical mass limit (e.g., van Kerkwijk et al. 1995; Barziv et al. 2001; Quaintrell et al. 2003; van der Meer et al. 2005; Özel et al. 2009; Güver et al. 2010).

The most precise measurements of NS masses are achieved by estimating the relativistic effects to orbital motion in binary systems. The exquisite precision of these mass measurements presents also a unique means to test general relativity in the "strong field" regime (e.g., Damour \& Taylor 1992; Psaltis 2008). As the masses of NSs also retain information about the past value of the effective gravitational constant $G$, with the determination of the NS mass range it may be even possible to probe the potential evolution of such physical constants (Thorsett 1996).

A comprehensive insight into the underlying mass distribution of NSs thus provides not only the means to study NS specific problems. It also offers diverse sets of constraints that can be as broad as the high-mass star formation history of the Galaxy (Gould 2000), or as particular as the compression modulus of symmetric nuclear matter (Glendenning 1986; Lattimer et al. 1990).

The present work aims to set up a framework by which we can probe the underlying mass distributions implied by radio pulsar observations. We develop a Bayesian framework that not only allows more flexibility for the inferred distribution but also accommodates asymmetric measurement errors in full parametric form. Unlike conventional statistical methods, with a Bayesian approach it is possible to separately infer peaks, shapes and cutoff values of the distribution with appropriate uncertainty quantification. This gives us unique leverage to probe these parameters which separately trace independent astrophysical and evolutionary processes.

In order to prevent contamination of the population, which may lead to systematic deviations from the probed mass distribution, we keep the observed pulsar sample as uniform as possible. We choose mass measurements that do not have strong a priori model dependencies and therefore can be considered secure.

In $\S 2$ we review theoretical constraints on NS masses. We derive useful quantities such as the NS birth mass $M_{\text {birth }}$, the amount of mass expected to be transferred onto the NS primary during recycling $\Delta m_{a c c}$, and the viable range of maximum mass (upper $97.5 \%$ probability) cutoff value $M_{\max }$ for NSs. The observations are reviewed in $\S 3$. We describe the statistical approach used to probe the underlying NS mass distribution in $\S 4$. After we summarize in $\S 5$, the range of implications and following conclusions are discussed in $\S 6$. For brevity, the details of the statistical model, the method for inference, and results from model checking are included in the Appendix $\S \mathrm{A}$ and $\S \mathrm{B}$.

\section{THEORETICAL CONSTRAINTS}

This section summarizes theoretical estimates on the birth mass of NSs, the amount of mass that can be accreted onto NSs, and the constraints on the maximum NS mass.

\subsection{Birth Mass}

The canonical mass limit $M_{c h} \sim 1.4 \mathrm{M}_{\odot}$ is the critical mass beyond which the degenerate remnant core of a massive star or a white dwarf will lose gravitational stability and collapse into a NS. This limiting mass is an approximation which is sensitive to several nuclear, relativistic and geometric effects (see Ghosh 2007; P. Haensel, A. Y. Potekhin, \& D. G. Yakovlev 2007, for review). In addition to these effects, the variety of evolutionary processes that produce NSs warrant a careful treatment.

A more precise parametrization of the Chandrasekhar mass is

$$
M_{c h}=5.83 Y_{e}^{2} \mathrm{M}_{\odot}
$$

where $Y_{e}=n_{p} /\left(n_{p}+n_{n}\right)$ is the electron fraction. A perfect neutron-proton equality $\left(n_{p}=n_{n}\right)$ with $Y_{e}=$ 0.50 yields a critical mass of

$$
M_{c h}=1.457 \mathrm{M}_{\odot} \text {. }
$$

However, we have a sufficiently good insight into the processes that affect $M_{c h}$. So, we can go beyond the idealized cases and estimate the remnant's expected initial mass more realistically.

The inclusion of more reasonable electron fractions $\left(Y_{e}<0.50\right)$ yields smaller values for $M_{c h}$. General relativistic implications, surface boundary pressure corrections, and the reduction of pressure due to non-ideal Coulomb interactions $\left(e^{-}-e^{-}\right.$repulsion, ion-ion repulsion and $e^{-}$-ion attraction) at high densities all reduce the upper limit of $M_{c h}$.

On the other hand, the electrons of the progenitor (i.e., white dwarf or the core of a massive star) material are not completely relativistic. This reduces the pressure leading to an increase in the amount of mass required to reach the gravitational potential to collapse the star. Finite entropy corrections and the effects of rotation will also enhance the stability for additional mass. These corrections, as a result, yield a higher upper limit for $M_{c h}$.

The level of impact on the birth masses due to some of these competing effects is not well constrained as the details of the processes are not well understood. An inclusion of the effects that are due to the diversity in the evolutionary processes alone requires a $\approx 20 \%$ correction (for a detailed numerical treatment see Butterworth \& Ipser 1975) and therefore implies a broader mass range, i.e. $M_{c h} \sim 1.17-1.75 \mathrm{M}_{\odot}$.

The measured masses, however, are the effective gravitational masses rather than a measure of the baryonic mass content. After applying the quadratic correction term $M_{\text {baryon }}-M_{\text {grav }} \approx 0.075 M_{\text {grav }}^{2}$ (Timmes et al. 1996), one can get

$$
M_{\text {birth }} \sim 1.08-1.57 \mathrm{M}_{\odot}
$$

as a viable range for gravitational NS masses which is believed to encapsulate the range of NS birth masses. 


\subsection{Accreted Mass}

There is considerable evidence that at least some millisecond pulsars have evolved from a first generation of NSs which have accumulated mass and angular momentum from their evolved companion (Alpar et al. 1982; Radhakrishnan \& Srinivasan 1982; Wijnands \& van der Klis 1998; Markwardt et al. 2002; Galloway et al. 2002, 2005). There is also a line of arguments that support the possibility of alternative evolutionary processes that may enrich the millisecond pulsar population (Bailyn \& Grindlay 1990; Kiziltan \& Thorsett 2009).

Possible production channels for isolated millisecond pulsars are mergers of compact primaries or accretion induced collapse (AIC). In the case where a NS is produced via AIC, the final mass configuration of the remnant is determined by the central density of the progenitor $(\mathrm{C}-\mathrm{O}$ or O-Ne white dwarf) and the speed at which the conductive deflagration propagates (Woosley \& Weaver 1992).

While there are uncertainties for the parameters that describe the ignition and flame propagation, a careful treatment of the physics that tune the transition of an accreting white dwarf yields a unique baryonic mass $M_{\text {baryon }} \approx 1.39 \mathrm{M}_{\odot}$ for the remnant which gives a gravitational mass of $M_{\text {grav }} \sim 1.27 \mathrm{M}_{\odot}$ for NSs produced via AIC (Timmes et al. 1996). There is indirect evidence that the occurrence rate of AICs can be significant (Bailyn \& Grindlay 1990).

The physics of these production channels are still not understood well enough to make quantitative predictions of the NS mass distribution produced via these processes. But we can estimate the mass required to spin NSs up to millisecond periods by using timescale and angular momentum arguments.

For low mass X-ray binaries (LMXBs) accreting at typical rates of $\dot{m} \sim 10^{-3} \dot{M}_{\mathrm{Edd}}$, the amount of mass accreted onto a NS in $10^{10} \mathrm{yr}$ is $\Delta m \approx 0.10 \mathrm{M}_{\odot}$. One can also estimate the amount of angular momentum required to spin the accreting progenitor up to velocities that equal the Keplerian velocity at the co-rotation radius. In order to transfer sufficient angular momentum $(L=I \times \omega)$ and spin up a normal pulsar $(R \approx 12 \mathrm{~km}$, $I \approx 1.4 \times 10^{45} \mathrm{~g} \mathrm{~cm}^{2}$ ) to millisecond periods, an additional mass of $\Delta m \approx 0.20 \mathrm{M}_{\odot}$ is required. Hence,

$$
\Delta m_{a c c} \approx 0.10-0.20 \mathrm{M}_{\odot}
$$

will be sufficient to recycle NS primaries into millisecond pulsars.

\subsection{Maximum Mass}

The mass and the composition of NSs are intricately related. One of the most important empirical clues that would lead to constraints on a wide range of physical processes is the maximum mass of NSs. For instance, secure constraints on the maximum mass provide insight into the range of viable EOSs for matter at supranuclear densities.

A first order theoretical upper limit can be obtained by numerically integrating the Oppenheimer-Volkoff equations for a low-density EOS at the lowest energy state of the nuclei (Baym et al. 1971). This yields an extreme upper bound to the maximum mass of a NS at $M_{\max } \sim 3.2 \mathrm{M}_{\odot}$ (Rhoades \& Ruffini 1974). Any com- pact star to stably support masses beyond this limit requires stronger short-range repulsive nuclear forces that stiffens the EOSs beyond the causal limit. For cases in which causality is not a requisite $(v \rightarrow \infty)$ an upper limit still exist in general relativity $\approx 5.2 \mathrm{M}_{\odot}$ that considers uniform density spheres (Shapiro \& Teukolsky 1983). However, for these cases the extremely stiff EOSs that require the sound speed to be super-luminal $\left(d P / d \rho \geq c^{2}\right)$ are considered non-physical.

Differentially rotating NSs that can support significantly more mass than uniform rotators can be temporarily produced by binary mergers (Baumgarte et al. 2000). While differential rotation provides excess radial stability against collapse, even for modest magnetic fields, magnetic braking and viscous forces will inevitably bring differentially rotating objects into uniform rotation (Shapiro 2000). Therefore, radio pulsars can be treated as uniform rotators when calculating the maximum NS mass.

While general relativity along with the causal limit put a strict upper limit on the maximum NS mass at $\sim 3.2 \mathrm{M}_{\odot}$, the lower bound is mostly determined by the still unknown EOS of matter at these densities and therefore is not well constrained. There are modern EOSs with detailed inclusions of nuclear processes such as kaon condensation and nucleon-nucleon scattering which affect the stiffness. These EOSs give a range of $1.5-2.2 \mathrm{M}_{\odot}$ as the lower bound for the maximum NS mass (Thorsson et al. 1994; Kalogera \& Baym 1996). Although these lower bounds for a maximum NS mass are implied for a variation of more realistic EOSs, it is still unclear whether any of these values are favored. Therefore,

$$
M_{\max } \sim 1.5-3.2 \mathrm{M}_{\odot}
$$

can be considered a secure range for the maximum NS mass value.

\section{OBSERVATIONS}

The timing measurements of radio pulsations from NSs offer a precise means to constrain orbital parameters (Manchester \& Taylor 1977). For systems where only five Keplerian orbital parameters (orbital period: $P_{b}$, projected semi-major axis: $x$, eccentricity: $e$, longitude and the time of periastron passage: $\left.\omega_{0}, T_{0}\right)$ are measured, individual masses of the primary $\left(m_{1}\right)$ and secondary $\left(m_{2}\right)$ stars, and the orbital inclination $i$ cannot be separately constrained. They remain instead related by the measured mass function $f$ which is given by

$$
f=\frac{\left(m_{2} \sin i\right)^{3}}{M^{2}}=\left(\frac{2 \pi}{P_{b}}\right)^{2} x^{3} \mathrm{~T}_{\odot}^{-1}
$$

where $M=m_{1}+m_{2}$ and masses are in solar units, the constant $\mathrm{T}_{\odot} \equiv \mathrm{G} \mathrm{M}_{\odot} / \mathrm{c}^{3}=4.925490947 \mu \mathrm{s}$, and $x$ is measured in light seconds.

For some binary systems, the timing residuals cannot be modeled with only Keplerian parameters when the effects of general relativity are measurable. In these cases, the gravitational influence can be parametrized as five potentially measurable post-Keplerian (PK) parameters which have similar interpretations (Taylor 1992); (1) $\dot{\omega}$ : advance of periastron $(2) \dot{P}_{b}$ : orbital period decay $(3)$ $\gamma$ : time dilation-gravitational redshift (4) $r$ : range of 
Table 1

Double neutron star systems

\begin{tabular}{|c|c|c|c|}
\hline Pulsar & Mass $\left[\mathrm{M}_{\odot}\right]$ & $68 \%$ central limits & Refs. $^{a}$ \\
\hline \multicolumn{4}{|c|}{ Double neutron star binaries } \\
\hline $\begin{array}{l}\text { J0737-3039 } \\
\text { pulsar A } \\
\text { pulsar B } \\
\text { total }\end{array}$ & $\begin{array}{l}1.3381 \\
1.2489 \\
2.58708\end{array}$ & $\begin{array}{l} \pm 0.0007 \\
\pm 0.0007 \\
\pm 0.00016\end{array}$ & {$[1]$} \\
\hline $\begin{array}{l}\text { J1518+4904 } \\
\text { pulsar } \\
\text { companion } \\
\quad \text { total }\end{array}$ & $\begin{array}{l}1.56 \\
1.05 \\
2.61\end{array}$ & $\begin{array}{l}+0.13 /-0.44 \\
+0.45 /-0.11 \\
\pm 0.070\end{array}$ & {$[2]$} \\
\hline $\begin{array}{l}\text { B1534+12 } \\
\text { pulsar } \\
\text { companion } \\
\quad \text { total }\end{array}$ & $\begin{array}{l}1.3332 \\
1.3452 \\
2.678428\end{array}$ & $\begin{array}{l} \pm 0.0010 \\
\pm 0.0010 \\
\pm 0.000018\end{array}$ & {$[3]$} \\
\hline $\begin{array}{l}\text { J1756-2251 } \\
\text { pulsar } \\
\text { companion } \\
\quad \text { total }\end{array}$ & $\begin{array}{l}1.40 \\
1.18 \\
2.574\end{array}$ & $\begin{array}{l}+0.02 /-0.03 \\
+0.03 /-0.02 \\
\pm 0.003\end{array}$ & {$[4]$} \\
\hline $\begin{array}{l}\text { J1811-1736 } \\
\text { pulsar } \\
\text { companion } \\
\quad \text { total }\end{array}$ & $\begin{array}{l}1.56 \\
1.12 \\
2.57\end{array}$ & $\begin{array}{l}+0.24 /-0.45 \\
+0.47 /-0.13 \\
\pm 0.10\end{array}$ & {$[5,6]$} \\
\hline $\begin{array}{l}\text { J1829+2456 } \\
\text { pulsar } \\
\text { companion } \\
\quad \text { total }\end{array}$ & $\begin{array}{l}1.20 \\
1.40 \\
2.59\end{array}$ & $\begin{array}{l}+0.12 /-0.46 \\
+0.46 /-0.12 \\
\pm 0.02\end{array}$ & {$[7]$} \\
\hline $\begin{array}{l}\text { J1906+0746 } \\
\text { pulsar } \\
\text { companion } \\
\quad \text { total }\end{array}$ & $\begin{array}{l}1.248 \\
1.365 \\
2.61\end{array}$ & $\begin{array}{l} \pm 0.018 \\
\pm 0.018 \\
\pm 0.02\end{array}$ & {$[8,9]$} \\
\hline $\begin{array}{l}\text { B1913+16 } \\
\text { pulsar } \\
\text { companion } \\
\quad \text { total }\end{array}$ & $\begin{array}{l}1.4398 \\
1.3886 \\
2.828378\end{array}$ & $\begin{array}{l} \pm 0.0002 \\
\pm 0.0002 \\
\pm 0.000007\end{array}$ & {$[10,11]$} \\
\hline $\begin{array}{l}\text { B2127+11C } \\
\text { pulsar } \\
\text { companion } \\
\quad \text { total }\end{array}$ & $\begin{array}{l}1.358 \\
1.354 \\
2.71279\end{array}$ & $\begin{array}{l} \pm 0.010 \\
\pm 0.010 \\
\pm 0.00013\end{array}$ & {$[12, \star]$} \\
\hline
\end{tabular}

a References: 1: Kramer et al. (2006), 2: Thorsett \& Chakrabarty (1999), 3: Stairs et al. (2002), 4: Faulkner et al. (2005), 5: Stairs (2006), 6: Corongiu et al. (2007), 7: Champion et al. (2005), 8: Kasian (2008), 9: Lorimer et al. (2006b), 10: Weisberg et al. (2010), 11: Taylor (1992), 12: Jacoby et al. (2006), $\star$ : In globular cluster.

Shapiro delay (5) $s$ : shape of Shapiro delay, where these are described by

$$
\begin{gathered}
\dot{\omega}=3\left(\frac{P_{b}}{2 \pi}\right)^{-5 / 3}\left(\mathrm{~T}_{\odot} M\right)^{2 / 3}\left(1-e^{2}\right)^{-1}, \\
\dot{P}_{b}=-\frac{192 \pi}{5}\left(\frac{P_{b}}{2 \pi}\right)^{-5 / 3}\left(1+\frac{73}{24} e^{2}+\frac{37}{96} e^{4}\right) \times \\
\left(1-e^{2}\right)^{-7 / 2} \mathrm{~T}_{\odot}^{5 / 3} m_{1} m_{2} M^{-1 / 3}, \\
\gamma=e\left(\frac{P_{b}}{2 \pi}\right)^{1 / 3} \mathrm{~T}_{\odot}^{2 / 3} M^{-4 / 3} m_{2}\left(m_{1}+2 m_{2}\right),
\end{gathered}
$$

Table 2

Neutron star - white dwarf binary systems

\begin{tabular}{lllr}
\hline \hline Pulsar & Mass $\left[\mathrm{M}_{\odot}\right]$ & $68 \%$ central limits & Refs. $^{\mathrm{a}}$ \\
\hline \multicolumn{3}{c}{ Neutron star - white dwarf binaries } \\
\hline J0437-4715 & 1.76 & \pm 0.20 & {$[1]$} \\
J0621+1002 & 1.70 & $+0.10 /-0.17$ & {$[2]$} \\
J0751+1807 & 1.26 & \pm 0.14 & {$[2]$} \\
J1012+5307 & 1.64 & \pm 0.22 & {$[3]$} \\
J1141-6545 & 1.27 & \pm 0.01 & {$[4]$} \\
J1614-2230 & 1.97 & \pm 0.04 & {$[5]$} \\
J1713+0747 & 1.53 & $+0.08 /-0.06$ & {$[6]$} \\
J1802-2124 & 1.24 & \pm 0.11 & {$[7]$} \\
B1855+09 & 1.57 & $+0.12 /-0.11$ & {$[8]$} \\
J1909-3744 & 1.438 & \pm 0.024 & {$[9]$} \\
B2303+46 & 1.38 & $+0.06 /-0.10$ & {$[10]$} \\
J0024-7204H & 1.48 & $+0.03 /-0.06$ & {$[\dagger, \star]$} \\
J0514-4002A & 1.49 & $+0.04 /-0.27$ & {$[\dagger, \star]$} \\
B1516+02B & 2.10 & \pm 0.19 & {$[\dagger, \star]$} \\
J1748-2446I & 1.91 & $+0.02 /-0.10$ & {$[\dagger, \star]$} \\
J1748-2446J & 1.79 & $+0.02 /-0.10$ & {$[\dagger, \star]$} \\
B1802-07 & 1.26 & $+0.08 /-0.17$ & {$[10, \star]$} \\
B1911-5958A & 1.40 & $+0.16 /-0.10$ & {$[11, \star]$} \\
\hline
\end{tabular}

a References: 1: Verbiest et al. (2008), 2: Nice et al. (2008), 3: Callanan et al. (1998), 4: Bhat et al. (2008), 5: Demorest et al. (2010) 6: Splaver et al. (2005), 7: $\quad$ Ferdman et al. (2010), 8: Nice et al. (2003), 9: Jacoby et al. (2005), 10: Thorsett \& Chakrabarty (1999), 11: Bassa et al. (2006), $\dagger$ : P. Freire (personal communication), $\star$ : In globular cluster.

$$
\begin{gathered}
r=\mathrm{T}_{\odot} m_{2}, \\
s=x\left(\frac{P_{b}}{2 \pi}\right)^{-2 / 3} \mathrm{~T}_{\odot}^{-1 / 3} M^{2 / 3} m_{2}^{-1} .
\end{gathered}
$$

A comprehensive review of the observational techniques and measurements can be found in Lorimer \& Kramer (2004) and Stairs (2006).

In systems where at least two PK parameters can be measured, $m_{1}$ and $m_{2}$ may be individually determined. In rare cases, more than two PK parameters are measurable. These over-constrained systems present a unique means to test for consistent strong-field gravitational theories (Taylor \& Weisberg 1989).

In Table 1 and Table 2 we compile a comprehensive list of NS masses that are determined by the relativistic orbital phenomena reflected onto the binary system orbital parameters. We include the mass estimates along with the $68 \%$ confidence limits which are plotted on Figure 1.

Pulsar surveys suffer from selection effects, especially in the low frequency $(<1 \mathrm{GHz})$ regime. Recent surveys in $1.4 \mathrm{GHz}$ have revealed a greater number of pulsars compared to previous surveys. The inverse square law, radio sky background, and propagation effects (i.e. pulse dispersion and scattering) in the interstellar medium also introduce global selection biases to the observed population. In addition to these biases affecting the population at large, there are also source dependent biases such as radio intermittency, pulse nulling, and the finite size of the emission beam. However, there is no evidence that 


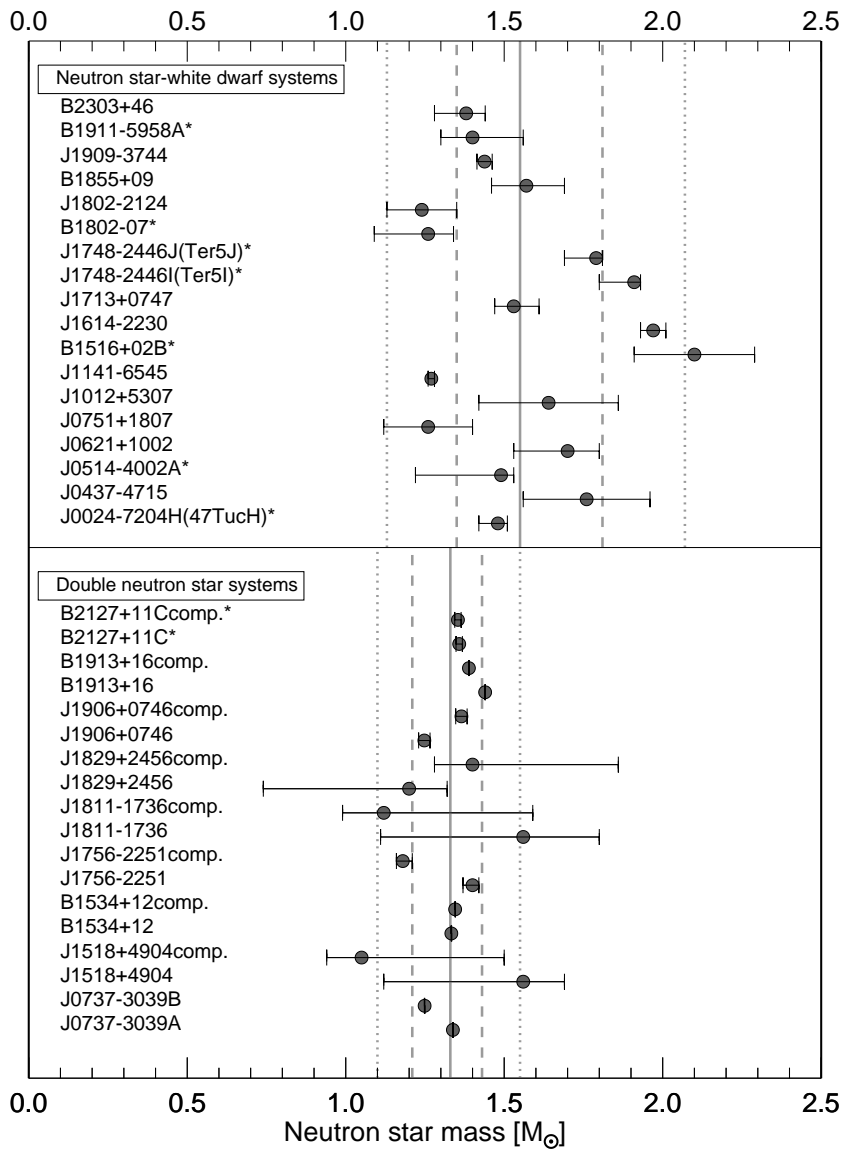

Figure 1. Measured masses of radio pulsars. All error bars indicate the central $68 \%$ confidence limits. Vertical solid lines are the peak values of the underlying mass distribution for DNS $\left(m=1.33 \mathrm{M}_{\odot}\right)$ and NS-WD $\left(m=1.55 \mathrm{M}_{\odot}\right)$ systems. The dashed and dotted vertical lines are the central $68 \%$ and $95 \%$ predictive probability intervals of the inferred mass distribution in Figure 2. " $\star$ " points to pulsars found in globular clusters.

these source dependent selection biases have mass dependent effects (see Lorimer et al. 2006a). Therefore, we do not expect that these selection effects introduce mass dependent biases to the observed distribution.

We aim to prevent possible contamination of the sample with sub-populations which may have gone through different and not well understood evolutionary paths (e.g., isolated NSs). Even for the better constrained formation processes that lead to the production of DNS and NS-WD systems, theoretical models estimating the final NS masses are tentative.

\section{ESTIMATING THE UNDERLYING MASS DISTRIBUTION}

Recent advances in statistical methods have reached a level which allows us to extract information from sparse data with unprecedented detail.

It can be clearly argued why modeling the underlying NS mass distribution as a single homogenous population is over-simplistic. There is no compelling line of reasoning that would require a single coherent (unimodal) mass distribution for NSs that we know have dissimilar evolutionary histories and possibly different production channels (e.g., see Podsiadlowski et al. 2004). In fact, there is an increasing number of measurements that show clear signatures for masses that deviate from the canonical value of $1.4 \mathrm{M}_{\odot}$. For instance, recent findings of van Kerkwijk et al. (2010) imply that the mass for PSR $\mathrm{B} 1957+20$ may be as high as $2.4 \pm 0.12 \mathrm{M}_{\odot}$. As we show in $§ 4.1$, a flexible modeling approach can be used to test whether the implied masses belong to the same distribution. We argue that with the number of NS mass measurements available in Table 1 and Table 2, clear signatures should be manifest in the inferred underlying mass distributions if appropriate statistical techniques are utilized. Since we still operate in the sparse data regime, it is useful, if not necessary, to use Bayesian inference methods.

For the range of calculations we use mass measurements obtained directly from pulsar timing. The methods used for estimating NS masses other than radio timing, have intrinsically different systematics, and therefore require a more careful treatment when assessing the implied NS mass distribution. The inclusion of mass estimates of NSs in X-ray binaries along with these more secure measurements would potentially perturb the homogeneity of the sample and the coherence of the inference.

For an all inclusive assessment of NS masses, more sophisticated hierarchical inference methods may be required. For sparse data, a proper statistical treatment of different systematic effects and a priori assumptions is not trivial. Also, the expected loss in precision may outweigh the gain obtained from a more detailed approach. Without properly tested and calibrated tools, further inclusion of NSs whose masses are not measured by pulsar timing in radio may just contaminate the sample and can therefore be misleading (e.g., see Steiner et al. 2010).

\subsection{Statistical Model}

Here, we present the statistical model to estimate the NS mass distribution. The approach is based on a formulation that incorporates errors in measurement of NS mass estimates. Specifically, the model formulation:

$$
m_{i}=\mathcal{M}_{i}+w_{i}, \quad i=1, \ldots, n
$$

where, for the $i$-th neutron star, $m_{i}$ is the estimate of the NS mass $\mathcal{M}_{i}$ and $w_{i}$ is the associated error. We thus need a model for the NS mass distribution and for the measurement error distribution. Evidently, the key focus of inference is the NS mass distribution, but a flexible specification for the error distribution is needed to ensure that this inference is not biased. At the same time, the model specification must take into account the limited amount of data. The proposed modeling approach achieves a balance between these considerations, and importantly, enables relatively straightforward implementation of inference through posterior simulation computational methods.

Visual inspection of the pulsar mass estimates (see Table 1, Table 2 and Figure 1) suggests that skewness may be present in the NS mass distribution, at least for the NS-WD systems. It is therefore important to extend the normality assumption which is implicit in the existing estimation methods. Furthermore, it is clear from the error bars of the pulsar mass estimates that an asymmetric measurement error distribution is needed for some of the observations, especially for the DNS systems. The statistical model developed below allows for skewness both 
in the NS mass distribution and the error distribution while encompassing the normal distribution for either as a special case.

The pulsars in less constrained systems (e.g., with only one post-Keplerian parameter determined) typically have asymmetric measurement errors. The flexibility of the statistical modeling approach developed here allows us to take full advantage of all available mass measurements in Table 1 and Table 2. It is noteworthy that the model is generic enough so it can be adopted to other similar astrophysical problems and serve as a useful reference.

Regarding the model for the NS mass distribution, we work with a skewed normal distribution with density function given by

$$
\mathrm{SN}(\mathcal{M} \mid \mu, \sigma, \alpha)=\frac{2}{\sigma} \phi\left(\frac{\mathcal{M}-\mu}{\sigma}\right) \Phi\left(\frac{(\mathcal{M}-\mu) \alpha}{\sigma}\right)
$$

where $\phi(\cdot)$ and $\Phi(\cdot)$ denote the density function and cumulative distribution function, respectively, of the standard normal distribution. Here, $\mu \in \mathbb{R}$ is a location parameter, $\sigma \in \mathbb{R}^{+}$is a scale parameter, and $\alpha \in \mathbb{R}$ is a skewness parameter. This model was studied by Azzalini (1985) and is one of the more commonly used skewed normal distributions. Note that $\alpha=0$ yields the normal distribution with mean $\mu$ and standard deviation $\sigma$ as a special case of (13), which highlights the role of $\alpha$ as a skewness parameter. In particular, positive/negative values of $\alpha$ result in right/left skewness for the density in (13). Hence, an appealing feature of this model is that, within the context of Bayesian inference, we can make probabilistic assessments for skewness of the NS mass distribution relative to a normal distribution through, for instance, a posterior interval estimate for parameter $\alpha$. As discussed in $\S 4.2$, we find some evidence for skewness in the NS mass distribution corresponding to the NS-WD systems, but not for the DNS systems.

Next, we describe the model for the error distribution, which is motivated by the process used to produce the pulsar mass estimates and the associated error bars. For each pulsar (either from a NS-WD or a DNS system) an empirical density curve for its mass is constructed based on how well the post-Keplerian parameters of the system can be constrained. We generically denote the final constructed density for the $i$-th pulsar as $h_{i}(m)$, and note that, although it is unimodal, it may be asymmetric (especially for pulsars that are in a system for which only one post-Keplerian parameter can be constrained) resulting in the asymmetric error bars reported for some of the systems in Table 1 and Table 2. The pulsar mass estimate, $m_{i}$, is obtained as the mode of this density, whereas the error bars, $+u_{i} /-\ell_{i}$, define the interval, $\left(m_{i}-\ell_{i}, m_{i}+u_{i}\right)$, of the smallest possible length with $68 \%$ probability coverage. Numerically, the interval is obtained by starting from the peak value of $h_{i}(m)$ and slicing down the density until $\int_{m_{i}-\ell_{i}}^{m_{i}+u_{i}} h_{i}(m) \mathrm{d} m=0.68$, which, given the unimodal shape of density $h_{i}(m)$, also implies that $h_{i}\left(m_{i}-\ell_{i}\right)=h_{i}\left(m_{i}+u_{i}\right)$.

Hence, in the context of model (12), the errors $w_{i}$ are realizations from a distribution with mode at 0 . We thus seek a measurement error distribution which is parameterized in terms of its mode, allows asymmetry around the mode, and yields the normal distribution in the special case of symmetry. A particularly suitable choice is the asymmetric normal distribution studied in Fernández \& Steel (1998) with density function given by

$$
\begin{aligned}
& \operatorname{AN}(w \mid c, d)= \\
& \frac{2}{d\left(c+\frac{1}{c}\right)}\left\{\phi\left(\frac{w}{c d}\right) 1_{[0, \infty)}(w)+\phi\left(\frac{c w}{d}\right) 1_{(-\infty, 0)}(w)\right\}
\end{aligned}
$$

where $c>0, d>0$, and $1_{A}(\cdot)$ denotes the indicator function of set $A$. The mode of this density is at 0 , when $c=1$ it reduces to the normal density with mean 0 and standard deviation $d$, and when $c>1(c<1)$ it is right skewed (left skewed). Therefore, $d$ plays the role of a scale parameter, whereas $c$ is the asymmetry parameter.

A practically important feature of the asymmetric normal density in (14) is that it leads to straightforward estimation of parameters $c_{i}$ and $d_{i}$ for the $i$-th pulsar, using the values of the $68 \%$ central limits, $+u_{i} /-\ell_{i}$, in Table 1 and Table 2. First, from the condition AN $\left(-\ell_{i} \mid c_{i}, d_{i}\right)=$ $\operatorname{AN}\left(u_{i} \mid c_{i}, d_{i}\right)$ we obtain $\phi\left(-c_{i} d_{i}^{-1} \ell_{i}\right)=\phi\left(c_{i}^{-1} d_{i}^{-1} u_{i}\right)$, which in turn yields $c_{i}=\left(u_{i} / \ell_{i}\right)^{1 / 2}$. Next, with $c_{i}$ determined, we specify $d_{i}$ by solving numerically for its value that satisfies $\int_{-\ell_{i}}^{u_{i}} \operatorname{AN}\left(w \mid c_{i}, d_{i}\right) \mathrm{d} w=0.68$. Computing this equation involves normal distribution function evaluations, and it can be easily shown that there is a unique solution for $d_{i}$. Note that $c_{i}=1$ when $\ell_{i}=u_{i}$, and thus the error distribution is normal for the pulsars with symmetric error bars. However, for pulsars with asymmetric error bars, asymmetry is introduced in the respective error distribution components. The extent of the asymmetry depends on the relative magnitude of $\ell_{i}$ and $u_{i}$; for instance, the maximum value for the asymmetry parameter $c_{i}$ is 2.02 corresponding to PSR J1518+4904's companion, and the minimum value is 0.38 corresponding to PSR J0514-4002A.

\subsection{Inferring the Neutron Star Mass Distribution}

For each of the NS-WD and DNS systems, the data vector comprises data $=\left\{\left(m_{i}, c_{i}, d_{i}\right): i=1, \ldots, n\right\}$, with $\left(c_{i}, d_{i}\right)$ computed as detailed in Section 4.1. Then, combining the models for the NS mass and error distributions in (13) and (14), respectively, the hierarchical model for the data can be written as

$$
\begin{aligned}
m_{i} \mid \mathcal{M}_{i} & \sim \operatorname{AN}\left(m_{i}-\mathcal{M}_{i} \mid c_{i}, d_{i}\right), \quad i=1, \ldots, n \\
\mathcal{M}_{i} \mid \mu, \sigma, \alpha & \sim \operatorname{SN}\left(\mathcal{M}_{i} \mid \mu, \sigma, \alpha\right), \quad i=1, \ldots, n
\end{aligned}
$$

That is, given the respective NS masses $\mathcal{M}_{i}$, the pulsar mass estimates $m_{i}$ arise conditionally independently from the asymmetric normal response distribution with mode at $\mathcal{M}_{i}$. Here, the $\mathcal{M}_{i}$ are modeled as conditionally independent realizations, given parameters $(\mu, \sigma, \alpha)$, from the skewed normal NS mass distribution.

Now, the likelihood function for the NS mass distribution parameters arises from the hierarchical model in (15) by marginalizing over the $\mathcal{M}_{i}$, that is,

$$
\begin{aligned}
& \mathcal{L}(\mu, \sigma, \alpha ; \text { data })= \\
& \prod_{i=1}^{n} \int \operatorname{AN}\left(m_{i}-\mathcal{M}_{i} \mid c_{i}, d_{i}\right) \operatorname{SN}\left(\mathcal{M}_{i} \mid \mu, \sigma, \alpha\right) \mathrm{d} \mathcal{M}_{i} .
\end{aligned}
$$

The integral is readily available analytically only in the special case of a normal distribution for both the errors and NS masses ( $c_{i}=1$ and $\alpha=0$, respectively). Therefore, in general, numerical maximization of the likelihood 


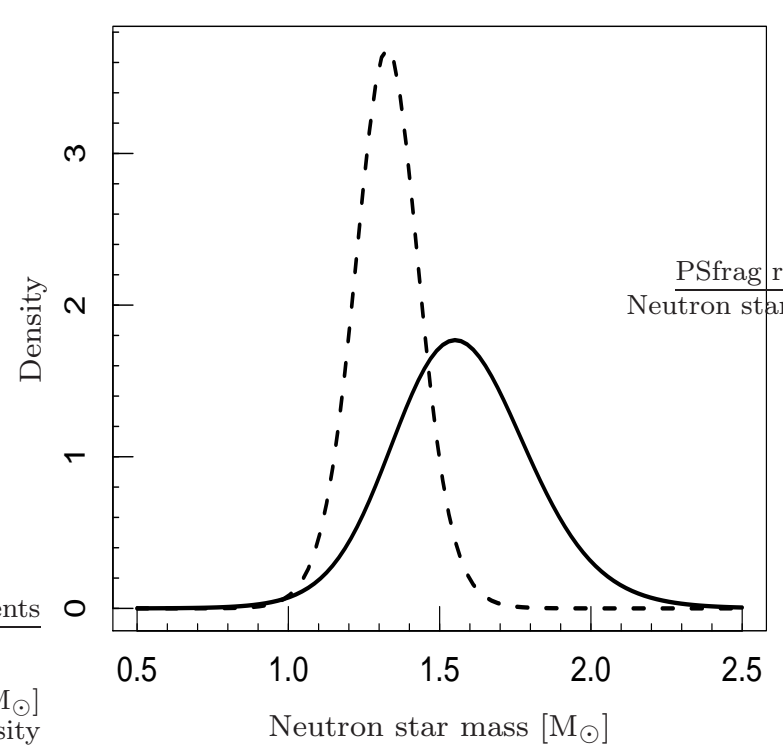

Figure 2. Posterior predictive density estimates for the NS mass distribution. DNS systems (dashed line) and NS-WD systems (solid line) mass densities have respective peaks at $1.33 \mathrm{M}_{\odot}$ and $1.55 \mathrm{M}_{\odot}$. The $68 \%$ and $95 \%$ posterior predictive intervals are given by $\left(1.21 \mathrm{M} \odot, 1.43 \mathrm{M}_{\odot}\right)$ and $\left(1.10 \mathrm{M} \odot, 1.55 \mathrm{M}_{\odot}\right)$ for the DNS systems, and by $\left(1.35 \mathrm{M}_{\odot}, 1.81 \mathrm{M}_{\odot}\right)$ and $\left(1.13 \mathrm{M}_{\odot}, 2.07 \mathrm{M}_{\odot}\right)$ for the NS-WD systems.

function to obtain the maximum likelihood estimates for parameters $\mu, \sigma$ and $\alpha$ is not straightforward. Even more challenging is uncertainty quantification for the point estimates, and its subsequent effect on the NS mass density; this would require large-sample asymptotic results the use of which is particularly problematic to justify given the small number of observations from both the DNS and NS-WD systems.

We thus employ the Bayesian approach to inference for the NS mass distribution, using Markov chain Monte Carlo (MCMC) methods for sampling from the posterior distribution of model parameters (Gelman et al. 2003). Under the Bayesian approach, model (15) is completed with priors for the NS mass distribution parameters. Details on the prior distributions are given in Appendix §A.

In addition to providing a coherent probabilistic framework for inference, the Bayesian model formulation enables harnessing the full hierarchical structure in (15). In particular, our posterior simulation method retains the individual NS masses $\mathcal{M}_{i}$ as part of the full parameter vector along with the NS mass distribution parameters $(\mu, \sigma, \alpha)$. In fact, we work with a transformed version $\left(\mu, \tau^{2}, \psi\right)$ of $(\mu, \sigma, \alpha)$, such that $\sigma^{2}=\tau^{2}+\psi^{2}$ and $\alpha=$ $\psi / \tau$, with $\tau>0$ and $\psi \in \mathbb{R}$. This re-parameterization facilitates implementation of the computational method for posterior inference as an efficient Gibbs sampler algorithm. Key in this direction is also a stochastic representation of the skewed normal distribution in (13) as a mixture of normal distributions (Henze 1986). The specific result is given in Appendix $\S \mathrm{A}$, which includes also the technical details of the MCMC posterior simulation method.

Implementing the Gibbs sampler described in Appendix $\S$ A, we obtain posterior samples for $\left(\mu, \tau^{2}, \psi\right)$ and thus, through the transformation discussed above, for parameters $(\mu, \sigma, \alpha)$. These samples can be used to explore
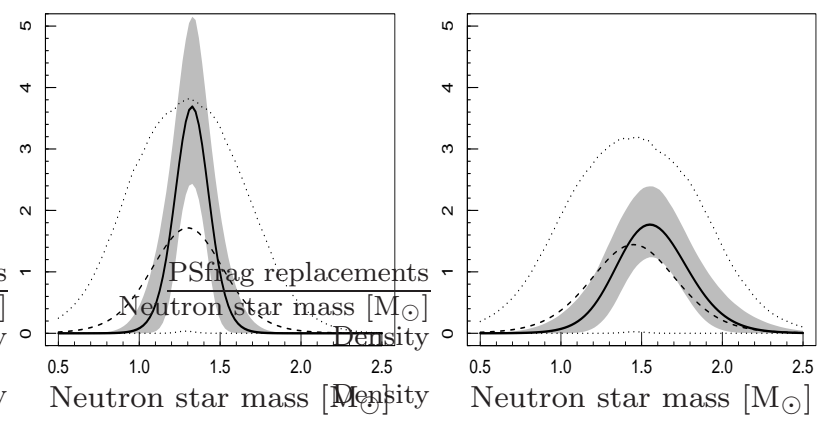

Figure 3. Comparison of prior mean and $95 \%$ interval estimates (dashed and dotted lines) with posterior mean and 95\% interval estimates (solid lines and gray bands) for the NS mass density corresponding to the DNS systems (left panel) and the NS-WD systems (right panel). Refer to $\S 4.2$ for further details.

a variety of inferences for the NS mass distribution.

First, the point estimate for the density of the NS mass distribution is given by the posterior predictive density, $\mathcal{P}\left(\mathcal{M}_{0} \mid\right.$ data), where $\mathcal{M}_{0}$ denotes the (unknown) mass of a "new" unobserved pulsar which we seek to estimate (predict) given the observed data. The posterior predictive density is given by

$$
\begin{aligned}
& \mathcal{P}\left(\mathcal{M}_{0} \mid \text { data }\right)= \\
& \int \mathrm{SN}\left(\mathcal{M}_{0} \mid \mu, \sigma, \alpha\right) p(\mu, \sigma, \alpha \mid \text { data }) \mathrm{d} \mu \mathrm{d} \sigma \mathrm{d} \alpha
\end{aligned}
$$

where $p(\mu, \sigma, \alpha \mid$ data $)$ denotes the posterior distribution of the model parameters. Using expression (16) along with the samples from $p(\mu, \sigma, \alpha \mid$ data $)$, we can compute through straightforward Monte Carlo integration the NS mass density estimates over any grid of mass values of interest. Figure 2 plots the posterior predictive NS mass density estimates for the DNS and NS-WD systems, which have peaks at $1.33 \mathrm{M}_{\odot}$ and $1.55 \mathrm{M}_{\odot}$, respectively. We can also sample from the posterior predictive distribution by sampling from the $\operatorname{SN}\left(\mathcal{M}_{0} \mid \mu, \sigma, \alpha\right)$ distribution (using its normal mixture stochastic representation) for each posterior sample of $(\mu, \sigma, \alpha)$. The resulting samples quantify posterior predictive uncertainty around the NS mass density peaks. In particular, for the DNS systems the $68 \%$ and $95 \%$ posterior predictive intervals are $\left(1.21 \mathrm{M}_{\odot}, 1.43 \mathrm{M}_{\odot}\right)$ and $\left(1.10 \mathrm{M}_{\odot}, 1.55 \mathrm{M}_{\odot}\right)$, whereas for the NS-WD systems the corresponding intervals are given by $\left(1.35 \mathrm{M}_{\odot}, 1.81 \mathrm{M}_{\odot}\right)$ and $\left(1.13 \mathrm{M}_{\odot}, 2.07 \mathrm{M}_{\odot}\right)$.

It is noteworthy from Figure 2 that the NS-WD systems posterior predictive density suggests positive skewness in the NS mass distribution. This is also reflected in the posterior distribution for the skewness parameter $\alpha$ given the NS-WD systems data; specifically, the posterior mean for $\alpha$ is 0.90 and $\operatorname{Pr}(\alpha>0 \mid$ data $)=0.78$. In contrast, the corresponding results for the DNS systems data are $\mathrm{E}(\alpha \mid$ data $)=-0.03$ and $\operatorname{Pr}(\alpha>0 \mid$ data $)=0.49$ supporting symmetry (and normality) for the DNS systems mass distribution.

Next, we supplement the point estimates in Figure 2 with uncertainty bands for the NS mass density. To this end, using a grid of mass values in $0.5 \mathrm{M}_{\odot}$ to $2.5 \mathrm{M}_{\odot}$, we evaluate the skewed normal NS mass density in (13) at each of the posterior samples for its parameters $(\mu, \sigma, \alpha)$. This produces a sample of densities which can be averaged to obtain the posterior mean NS mass density 
estimate, given by the solid lines in Figure 3. (Formally, the posterior mean estimate is equivalent to the posterior predictive density, and thus the solid lines in Figure 3 agree with the estimates in Figure 2.) However, we can now also depict the posterior uncertainty for the entire NS mass density through percentiles from the posterior sample of densities. In Figure 3, we use the 0.025 and 0.975 percentiles, and thus the gray bands depicting the posterior uncertainty correspond to $95 \%$ interval estimates for the NS mass density.

Finally, Figure 3 plots also the prior point and $95 \%$ interval estimates for the NS mass density. These are produced as discussed above for the posterior inference results, but in this case using samples from the prior distribution assigned to the model parameters. Hence, Figure 3 shows the prior guess at the NS mass density (the prior mean estimate given by the dashed line) as well as the extent of variability in the prior (encapsulated by the dotted lines). This provides an effective means to summarize the extent of prior information for the NS mass density incorporated into the model through the specific priors for the model parameters. Moreover, the comparison with the corresponding estimates given the data illustrates the amount of prior-to-posterior learning, which is evidently significant for both the DNS and NSWD systems.

\subsection{Model Checking}

The predictive performance of the statistical model developed in Sections 4.1 and 4.2 was evaluated using a well-founded technique for Bayesian model checking (see, for example, Chapter 6 of Gelman et al. 2003). Briefly, for each data point $\left(m_{i}, c_{i}, d_{i}\right)$, we obtained the posterior predictive distribution, $\mathcal{P}\left(m_{i}^{r e p} \mid\right.$ data), for replicated response $m_{i}^{r e p}$, that is, the pulsar mass estimate that we would observe if the experiment that produced the data was to be replicated. Details on sampling from these posterior predictive distributions are included in Appendix $\S \mathrm{B}$. An indication of how well the model is performing predictively can be obtained by checking where the observed pulsar mass estimate $m_{i}$ lies within the corresponding posterior predictive density. A relatively large number of observations falling in the tails of the respective predictive densities is indicative of lack of model fit. Under the proposed model, all pulsar mass estimates from both the DNS and NS-WD systems were effectively captured within their corresponding posterior predictive distributions; see the plots in Appendix $\S \mathrm{B}$. These results provide a further illustration of the predictive power of the model as it replicates the appropriate type of asymmetry for the responses with asymmetric measurement errors.

\section{SUMMARY}

We overview the physical processes that tune masses of NSs in $\S 2$. In order to theoretically estimate the viable range for NS masses, we derive the birth mass $(\S 2.1$, $\left.M_{\text {birth }}=1.08-1.57 \mathrm{M}_{\odot}\right)$ and the amount of mass expected to be transferred onto recycled NSs during the binary phase $\left(\S 2.2, \Delta m_{a c c} \approx 0.1-0.2 \mathrm{M}_{\odot}\right)$. We then discuss why the constraints on the maximum NS mass $\left(M_{\max }=1.5-3.2 \mathrm{M}_{\odot}\right)$ are less stringent and comment on the sources of uncertainties in $\S 2.3$.
In order to maintain a uniform approach in our analysis, we refrained from including additional constraints that may arise from assumptions such as the possible relationship between the binary period and the mass of the remnant white dwarf (i.e., $P_{b}-m_{2}$ relationship) suggested by Rappaport et al. (1995). While more elaborate and hierarchical implementation methods may be utilized in deducing implication of other assumptions, a use of more inclusive approaches may only convolute the mass inference, which is contrary to the goal of this work. Throughout our analysis, we only assume that Einstein's prescription for general relativity is correct and include mass measurements which are considered secure (§3).

We then subject the pulsar mass measurements to a detailed statistical analysis. In $\S 4$ we show that a Bayesian approach offers an effective means for inference (for detailed derivation, see Appendix $\S \mathrm{A}$ ), using a model that accommodates skewness in distributions for both the underlying NS masses and the measurement errors.

\section{DISCUSSION AND CONCLUSIONS}

\subsection{Previous Studies}

The first article that reviewed pulsar mass measurements in order to deduce the range of masses NSs can attain, was published by Joss \& Rappaport (1976). They used mass measurements from 5 sources (PSR B1913+16, Her X-1, Cen X-3, SMC X-1, and 3U0900-40), which were predominantly X-ray sources, and found a marginally consistent range of $1.4-1.8 \mathrm{M}_{\odot}$.

Finn (1994) used a Bayesian statistical approach for the first time to infer limits on the NS mass distribution. By using the mass measurements of 4 radio pulsars (PSRs B1913+16, B1534+12, B2127+11C, and B2303+46), he concluded that NS masses should fall mainly in the range between $1.3-1.6 \mathrm{M}_{\odot}$.

A comprehensive paper on pulsar masses was published by Thorsett \& Chakrabarty (1999). Their analysis based on 26 sources yielded a remarkably tight mass range at $1.38_{-0.10}^{+0.06} \mathrm{M}_{\odot}$. The width of their NS mass inference was mainly driven by the narrow error bands of the DNS mass measurements.

The recent work by Schwab et al. (2010) analyzes masses of 14 sources with an approach based on comparing the cumulative distribution function (CDF) with an idealized Gaussian. It is well understood that the K-S test should be used with caution in cases where deviations occur in the tails (Mason \& Schuenemeyer 1983). Additionally, even in data samples where the number of outliers in the tails are considerably larger and associated measurement errors are taken into account, a K-S approach will still remain limited in quantifying the significance of the outliers. Therefore, while the bimodal feature found for the initial mass function (i.e. $M_{b i r t h}$ ) may be consistent with theoretical expectations for remnant masses produced by electron-capture versus Fe-core collapse SNe (Podsiadlowski et al. 2004), the evidence for a deviation of $M_{\text {birth }}$ from a unimodal distribution is still tentative. In order to firmly establish a potential multi-modal feature for the NS birth mass distribution, a more diverse sample tested with statistics that allows sensitivity and performance checks are required.

\subsection{Maximum Mass Limit}


Our model is flexible and sensitive enough for detecting signatures of a potential truncation in the underlying mass distribution. We pay particular attention to whether there are signatures of truncation in the implied mass distribution at the high mass end for two reasons: 1) A high mass limit set by the EOS of the NS matter, or by general relativity, should produce a relatively sharp cut off, which will manifest itself as a truncation. 2) The nature of potential skewness of the underlying mass distribution will provide insight into how the NSs are produced and the evolutionary link between the two NS populations.

The masses of NSs in DNS systems imply a tight symmetric distribution while the distribution of masses of NSs in NS-WD systems show evidence of skewness with a heavy tail on the high mass end. On the other hand, both populations show no evidence of a strong truncation limit on either end.

These have important implications: The stochastic nature of evolutionary processes such as long term stable accretion naturally produces a wider distribution for NSs in NS-WD systems. This along with the lack of a strong truncation indicate that, in particular, the high mass end of the NS mass distribution is driven by evolutionary constraints. As a result, this rules out the possibility that an upper mass limit is set by the EOS of matter or general relativity for $\mathrm{NSs}$ with masses $\mathrm{M} \lesssim 2.1 \mathrm{M}_{\odot}$. Therefore, the $2.1 \mathrm{M}_{\odot}$ upper mass limit implied by NSs in NS-WD systems should be considered as a minimum secure limit to the maximum NS mass rather than an absolute upper limit to NS masses. The heavy tail of the mass distribution of NSs in NS-WD systems favors the possibility that at least some of these pulsars are born as massive NSs.

\subsection{Central Density and the Equation of State}

All EOSs that require a maximum NS mass $M_{\max } \leq$ $2.1 \mathrm{M}_{\odot}$ are ruled out. The implied stiffness of the EOS largely precludes the presence of meson condensates and hyperons at supranuclear densities. Consequently, lower central densities, larger radii and thicker crusts for NSs are favored (Shapiro \& Teukolsky 1983).

The energy density-radius relation implied by Tolman (1939), when combined with the causality limit, gives an analytical solution for an upper limit on the central density

$$
\rho_{c} M^{2}=15.3 \times 10^{15} \mathrm{M}_{\odot}^{2} \mathrm{~g} \mathrm{~cm}^{-3} .
$$

With a $2.1 \mathrm{M}_{\odot}$ secure lower limit on the maximum NS mass, we set a $95 \%$ confidence upper limit to the central density of NSs, which is

$$
\rho_{\max }<3.47 \times 10^{15} \mathrm{~g} \mathrm{~cm}^{-3}
$$

corresponding to $\approx 11 \rho_{s}$ for a fiducial saturation threshold $n_{s} \sim 0.16 \mathrm{fm}^{-3}$.

Exotic matter such as hyperons and Bose condensates significantly reduce the maximum mass of NSs. Therefore, a strict lower limit on the maximum NS mass $\mathrm{M}_{\max }>2.1 \mathrm{M}_{\odot}$ rules out soft EOSs with extreme low density softening and require the existence of exotic hadronic matter (see Lattimer \& Prakash 2007, for review). NSs with deconfined strange quark matter mostly have maximum predicted masses lower than $2.1 \mathrm{M}_{\odot}$. Hence, EOSs with strange quark matter that predict maximum masses smaller than $2.1 \mathrm{M}_{\odot}$ can also be ruled out as viable configurations for NS matter.

\subsection{Evidence for Alternative Evolution and the Formation of Massive Neutron Stars?}

A $2.1 \mathrm{M}_{\odot}$ upper limit to masses of NSs in NS-WD system poses a problem. If all millisecond pulsars were indeed NSs that are recycled from a first generation of normal pulsars, the implied distribution should be consistent with a recycled version of the initial mass distribution. While the peaks of the distributions for DNS and NS-WD systems appear to be consistent with the expectations of standard recycling $(\S 2.2)$, the widths imply otherwise. As shown in Figure 1, $\Delta m_{a c c}=0.22 \mathrm{M}_{\odot}$ lies within the expected range. However, with typical accretion rates experienced during the LMXB phase $\left(\dot{m}_{a c c} \sim 10^{-3} \dot{\mathrm{M}}_{\mathrm{Edd}}\right)$, it is difficult to accumulate sufficient mass onto NSs that started their lives as a $\sim 1.4 \mathrm{M}_{\odot}$ NS and produce NSs with masses $\sim 2.1 \mathrm{M}_{\odot}$ such as PSR B1516+02B. Even with initial masses of $\sim 1.6 \mathrm{M}_{\odot}$ these sources need to accrete $\Delta m \approx 0.4-0.5 \mathrm{M}_{\odot}$ during their active accretion phase. This requires long term stable active accretion at unusually high rates.

Based on the P-P demographics of millisecond pulsars, Kiziltan \& Thorsett (2009) argue that $\approx 30 \%$ of the millisecond pulsar population may be produced via a nonstandard evolutionary channel. This prediction falls in line with a distribution that has a consistent recycled peak but has an unusual width (and some skewness with a heavy tail on the high mass end) which extends up to $2.1 \mathrm{M}_{\odot}$. While it is difficult to quantify the formation rate(s) of non-standard processes that may produce these NSs, it is clear that the standard scenario requires at least a revision. Such a revision should consistently reconcile for the observed $\mathrm{P}-\dot{\mathrm{P}}$ distribution of millisecond pulsars, along with the long term sustainability of unusually high accretion rates that is required to produce the second generation of massive NSs.

The only viable alternative to a major revision of the mass evolution implied by the standard recycling scenario, also corroborated by the lack of truncation of the underlying NS mass distribution and enforced by the heavier tail of the skewed distribution inferred from NSWD systems, is then to form massive NSs.

The authors thank P. Freire for sharing updated pdf's from which some of the NS mass estimates were extracted in Table 2. B.K. and S.E.T. acknowledge NSF grant AST-0506453. The authors thank the anonymous referee for a critical review. After this work was submitted for initial review, Özel et al. (2012) discussed an alternate approach for estimating the NS mass distribution assuming a Gaussian underlying distribution.

\section{REFERENCES}

Alpar, M. A., Cheng, A. F., Ruderman, M. A., \& Shaham, J. 1982, Nature, 300, 728

Azzalini, A. 1985, Scandinavian Journal of Statistics, 12, 171

Baade, W. \& Zwicky, F. 1934a, Proceedings of the National

Academy of Science, 20, 259

- 1934b, Proceedings of the National Academy of Science, 20, 254

-. 1934c, Physical Review, 46, 76

Bailyn, C. D. \& Grindlay, J. E. 1990, ApJ, 353, 159 
Barziv, O., Kaper, L., Van Kerkwijk, M. H., Telting, J. H., \& Van Paradijs, J. 2001, A\&A, 377, 925

Bassa, C. G., van Kerkwijk, M. H., Koester, D., \& Verbunt, F. 2006, A\&A, 456, 295

Baumgarte, T. W., Shapiro, S. L., \& Shibata, M. 2000, ApJ, 528, L29

Baym, G., Pethick, C., \& Sutherland, P. 1971, ApJ, 170, 299

Bhat, N. D. R., Bailes, M., \& Verbiest, J. P. W. 2008, Phys. Rev. D, 77, 124017

Bombaci, I. 1996, A\&A, 305, 871

Butterworth, E. M. \& Ipser, J. R. 1975, ApJ, 200, L103

Callanan, P. J., Garnavich, P. M., \& Koester, D. 1998, MNRAS, 298,207

Chadwick, J. 1932, Nature, 129, 312

Champion, D. J., Lorimer, D. R., McLaughlin, M. A., Xilouris,

K. M., Arzoumanian, Z., Freire, P. C. C.. Lommen, A. N., Cordes, J. M., \& Camilo, F. 2005, MNRAS, 363, 929

Champion, D. J., Ransom, S. M., Lazarus, P., Camilo, F., Bassa, C., Kaspi, V. M., Nice, D. J., Freire, P. C. C.. Stairs, I. H., van Leeuwen, J., Stappers, B. W., Cordes, J. M., Hessels, J. W. T. Lorimer, D. R., Arzoumanian, Z., Backer, D. C., Bhat, N. D. R., Chatterjee, S., Cognard, I., Deneva, J. S., Faucher-Giguère, C., Gaensler, B. M., Han, J., Jenet, F. A., Kasian, L., Kondratiev, V. I., Kramer, M., Lazio, J., McLaughlin, M. A., Venkataraman, A., \& Vlemmings, W. 2008, Science, 320, 1309

Chandrasekhar, S. 1931, ApJ, 74, 81

Cook, G. B., Shapiro, S. L., \& Teukolsky, S. A. 1994, ApJ, 424, 823

Corongiu, A., Kramer, M., Stappers, B. W., Lyne, A. G., Jessner, A., Possenti, A., D'Amico, N., \& Löhmer, O. 2007, A\&A, 462, 703

Damour, T. \& Taylor, J. H. 1992, Phys. Rev. D, 45, 1840

Demorest, P. B., Pennucci, T., Ransom, S. M., Roberts, M. S. E. \& Hessels, J. W. T. 2010, Nature, 467, 1081

Faulkner, A. J., Kramer, M., Lyne, A. G., Manchester, R. N., McLaughlin, M. A., Stairs, I. H., Hobbs, G., Possenti, A., Lorimer, D. R., D'Amico, N., Camilo, F., \& Burgay, M. 2005, ApJ, 618, L119

Ferdman, R. D., Stairs, I. H., Kramer, M., McLaughlin, M. A., Lorimer, D. R., Nice, D. J., Manchester, R. N., Hobbs, G., Lyne, A. G., Camilo, F., Possenti, A., Demorest, P. B., Cognard, I., Desvignes, G., Theureau, G., Faulkner, A., \& Backer, D. C. 2010, ApJ, 711, 764

Fernández, C. \& Steel, F. 1998, Journal of the American Statistical Association, 93, 359

Finn, L. S. 1994, Physical Review Letters, 73, 1878

Freire, P. C. C. 2008, in American Institute of Physics Conference Series, Vol. 983, 40 Years of Pulsars: Millisecond Pulsars, Magnetars and More, ed. C. Bassa, Z. Wang, A. Cumming, \& V. M. Kaspi, 459-463

Freire, P. C. C., Ransom, S. M., Bégin, S., Stairs, I. H., Hessels, J. W. T., Frey, L. H., \& Camilo, F. 2008a, ApJ, 675, 670 Freire, P. C. C., Wolszczan, A., van den Berg, M., \& Hessels, J. W. T. 2008b, ApJ, 679, 1433

Fryer, C. L. \& Kalogera, V. 2001, ApJ, 554, 548

Galloway, D. K., Chakrabarty, D., Morgan, E. H., \& Remillard, R. A. 2002, ApJ, 576, L137

Galloway, D. K., Markwardt, C. B., Morgan, E. H., Chakrabarty, D., \& Strohmayer, T. E. 2005, ApJ, 622, L45

Gelman, A., Carlin, J., Stern, H., \& Rubin, D. 2003, Bayesian Data Analysis, Second Edition (Chapman \& Hall/CRC)

Ghosh, P. 2007, Rotation and Accretion Powered Pulsars (World Scientific Publishing Co)

Glendenning, N. K. 1986, Physical Review Letters, 57, 1120

Gould, A. 2000, ApJ, 535, 928

Güver, T., Özel, F., Cabrera-Lavers, A., \& Wroblewski, P. 2010 ApJ, 712, 964

Haensel, P. 2003, in EAS Publications Series, Vol. 7, EAS Publications Series, ed. C. Motch \&amp; J.-M. Hameury, 249

Heiselberg, H. \& Pandharipande, V. 2000, Annual Review of Nuclear and Particle Science, 50, 481

Henze, N. 1986, Scandinavian Journal of Statistics, 13, 271

Jacoby, B. A., Cameron, P. B., Jenet, F. A., Anderson, S. B., Murty, R. N., \& Kulkarni, S. R. 2006, ApJ, 644, L113

Jacoby, B. A., Hotan, A., Bailes, M., Ord, S., \& Kulkarni, S. R. 2005, ApJ, 629, L113

Joss, P. C. \& Rappaport, S. A. 1976, Nature, 264, 219

Kalogera, V. \& Baym, G. 1996, ApJ, 470, L61

Kasian, L. 2008, in American Institute of Physics Conference Series, Vol. 983, 40 Years of Pulsars: Millisecond Pulsars, Magnetars and More, ed. C. Bassa, Z. Wang, A. Cumming, \& V. M. Kaspi, 485-487

Kiziltan, B. \& Thorsett, S. E. 2009, ApJ, 693, L109
Kramer, M., Stairs, I. H., Manchester, R. N., McLaughlin, M. A., Lyne, A. G., Ferdman, R. D., Burgay, M., Lorimer, D. R. Possenti, A., D'Amico, N., Sarkissian, J. M., Hobbs, G. B. Reynolds, J. E., Freire, P. C. C., \& Camilo, F. 2006, Science, 314,97

Landau, L. D. 1932, Phys. Z. Sowjetunion, 1, 285

Lattimer, J. M. \& Prakash, M. 2004, Science, 304, 536

-. 2007, Phys. Rep., 442, 109

Lattimer, J. M., Prakash, M., Masak, D., \& Yahil, A. 1990, ApJ, 355,241

Lorimer, D. R., Faulkner, A. J., Lyne, A. G., Manchester, R. N., Kramer, M., McLaughlin, M. A., Hobbs, G., Possenti, A., Stairs, I. H., Camilo, F., Burgay, M., D'Amico, N., Corongiu, A., \& Crawford, F. 2006a, MNRAS, 372, 777

Lorimer, D. R. \& Kramer, M. 2004, Handbook of Pulsar Astronomy (Cambridge: Cambridge University Press)

Lorimer, D. R., Stairs, I. H., Freire, P. C., Cordes, J. M., Camilo, F., Faulkner, A. J., Lyne, A. G., Nice, D. J., Ransom, S. M., Arzoumanian, Z., Manchester, R. N., Champion, D. J., van Leeuwen, J., Mclaughlin, M. A., Ramachandran, R., Hessels, J. W., Vlemmings, W., Deshpande, A. A., Bhat, N. D., Chatterjee, S., Han, J. L., Gaensler, B. M., Kasian, L., Deneva, J. S., Reid, B., Lazio, T. J., Kaspi, V. M., Crawford, F. Lommen, A. N., Backer, D. C., Kramer, M., Stappers, B. W., Hobbs, G. B., Possenti, A., D'Amico, N., \& Burgay, M. 2006b, ApJ, 640, 428

Manchester, R. N. \& Taylor, J. H. 1977, Pulsars. (San Francisco, CA)

Markwardt, C. B., Swank, J. H., Strohmayer, T. E., in 't Zand, J. J. M., \& Marshall, F. E. 2002, ApJ, 575, L21

Mason, D. M. \& Schuenemeyer, J. H. 1983, The Annals of Statistics, 11, pp. 933

Nice, D. J., Splaver, E. M., \& Stairs, I. H. 2003, in Astronomical Society of the Pacific Conference Series, Vol. 302, Radio Pulsars, ed. M. Bailes, D. J. Nice, \& S. E. Thorsett, 75

Nice, D. J., Stairs, I. H., \& Kasian, L. E. 2008, in American Institute of Physics Conference Series, Vol. 983, 40 Years of Pulsars: Millisecond Pulsars, Magnetars and More, ed. C. Bassa, Z. Wang, A. Cumming, \& V. M. Kaspi, 453-458

Oppenheimer, J. R. \& Volkoff, G. M. 1939, Physical Review, 55, 374

Özel, F., Güver, T., \& Psaltis, D. 2009, ApJ, 693, 1775

Özel, F., Psaltis, D., Narayan, R., \& Santos Villarreal, A. 2012, ApJ, 757, 55

P. Haensel, A. Y. Potekhin, \& D. G. Yakovlev, ed. 2007, Astrophysics and Space Science Library, Vol. 326, Neutron Stars 1: Equation of State and Structure

Podsiadlowski, P., Langer, N., Poelarends, A. J. T., Rappaport, S., Heger, A., \& Pfahl, E. 2004, ApJ, 612, 1044

Psaltis, D. 2008, Living Reviews in Relativity, 11, 9

Quaintrell, H., Norton, A. J., Ash, T. D. C., Roche, P., Willems, B., Bedding, T. R., Baldry, I. K., \& Fender, R. P. 2003, A\&A, 401, 313

Radhakrishnan, V. \& Srinivasan, G. 1982, Current Science, 51, 1096

Ransom, S. M., Hessels, J. W. T., Stairs, I. H., Freire, P. C. C., Camilo, F., Kaspi, V. M., \& Kaplan, D. L. 2005, Science, 307, 892

Rappaport, S., Podsiadlowski, P., Joss, P. C., Di Stefano, R., \& Han, Z. 1995, MNRAS, 273, 731

Rhoades, C. E. \& Ruffini, R. 1974, Physical Review Letters, 32, 324

Schwab, J., Podsiadlowski, P., \& Rappaport, S. 2010, ApJ, 719, 722

Shapiro, S. L. 2000, ApJ, 544, 397

Shapiro, S. L. \& Teukolsky, S. A. 1983, Black holes, white dwarfs, and neutron stars: The physics of compact objects (John Wiley \& Sons, Inc.)

Splaver, E. M., Nice, D. J., Stairs, I. H., Lommen, A. N., \& Backer, D. C. 2005, ApJ, 620, 405

Stairs, I. H. 2006, Journal of Physics G Nuclear Physics, 32, 259

Stairs, I. H., Thorsett, S. E., Taylor, J. H., \& Wolszczan, A. 2002, ApJ, 581, 501

Steiner, A. W., Lattimer, J. M., \& Brown, E. F. 2010, ApJ, 722 33

Taylor, J. H. 1992, Royal Society of London Philosophical Transactions Series A, 341, 117

Taylor, J. H. \& Weisberg, J. M. 1989, ApJ, 345, 434

Thorsett, S. E. 1996, Physical Review Letters, 77, 1432

Thorsett, S. E. \& Chakrabarty, D. 1999, ApJ, 512, 288

Thorsson, V., Prakash, M., \& Lattimer, J. M. 1994, Nuclear Physics A, 572, 693

Timmes, F. X., Woosley, S. E., \& Weaver, T. A. 1996, ApJ, 457, 834

Tolman, R. C. 1939, Phys. Rev., 55, 364 
van der Meer, A., Kaper, L., van Kerkwijk, M. H., \& van den

Heuvel, E. P. J. 2005, in American Institute of Physics

Conference Series, Vol. 797, Interacting Binaries: Accretion,

Evolution, and Outcomes, ed. L. Burderi, L. A. Antonelli,

F. D'Antona, T. di Salvo, G. L. Israel, L. Piersanti,

A. Tornambè, \&amp; O. Straniero, 623-626

van Kerkwijk, M. H., Breton, R., \& Kulkarni, S. R. 2010, ArXiv e-prints:1009.5427

van Kerkwijk, M. H., van Paradijs, J., \& Zuiderwijk, E. J. 1995,

A\&A, 303, 497
Verbiest, J. P. W., Bailes, M., van Straten, W., Hobbs, G. B., Edwards, R. T., Manchester, R. N., Bhat, N. D. R., Sarkissian, J. M., Jacoby, B. A., \& Kulkarni, S. R. 2008, ApJ, 679, 675 Weisberg, J. M., Nice, D. J., \& Taylor, J. H. 2010, ApJ, 722, 1030 Wijnands, R. \& van der Klis, M. 1998, Nature, 394, 344

Woosley, S. E. \& Weaver, T. A. 1992, in Structure and Evolution of Neutron Stars, ed. D. Pines, R. Tamagaki, \& S. Tsuruta, 235

\section{APPENDIX}

\section{A. BAYESIAN INFERENCE FOR THE NEUTRON STAR MASS DISTRIBUTION}

\section{A.1. Hierarchical Model Formulation}

The skewed normal distribution in (13) admits a stochastic representation as a mixture of normal distributions with a truncated normal mixing distribution. Introducing truncated normal latent random variables $z_{i}$ and reparameterizing $\mathrm{SN}(\cdot \mid \mu, \sigma, \alpha)$ to $\mathrm{SN}\left(\cdot \mid \mu, \tau^{2}, \psi\right)$ as described in $\S 4.2$, we have

$$
\mathcal{M}_{i} \mid z_{i}, \mu, \tau^{2}, \psi \stackrel{\text { ind }}{\sim} \mathrm{N}\left(\mathcal{M}_{i} \mid \mu+\psi z_{i}, \tau^{2}\right) ; \quad z_{i} \stackrel{\text { ind }}{\sim} \mathrm{N}(0,1) 1_{[0, \infty)}\left(z_{i}\right)
$$

as a hierarchical mixture representation of $\mathrm{SN}\left(\mathcal{M}_{i} \mid \mu, \tau^{2}, \psi\right)$ (that is, if we marginalize over the $z_{i}$ in (A1), we obtain $\left.\mathcal{M}_{i} \mid \mu, \tau^{2}, \psi \stackrel{\text { ind }}{\sim} \mathrm{SN}\left(\mathcal{M}_{i} \mid \mu, \tau^{2}, \psi\right)\right)$. Here, $\mathrm{N}(0,1) 1_{[0, \infty)}\left(z_{i}\right)$ indicates a standard normal distribution restricted to $\mathbb{R}^{+}$.

To facilitate MCMC posterior simulation, we work with this formulation for the skewed normal distribution. The second line of the hierarchical model for the data given in (15) is therefore changed as indicated in (A1), to give the following hierarchical model for the data:

$$
\begin{gathered}
m_{i} \mid \mathcal{M}_{i} \stackrel{i n d}{\sim} \mathrm{AN}\left(m_{i}-\mathcal{M}_{i} \mid c_{i}, d_{i}\right), \quad i=1, \ldots, n \\
\mathcal{M}_{i} \mid z_{i}, \mu, \tau^{2}, \psi \stackrel{\text { ind }}{\sim} \mathrm{N}\left(\mathcal{M}_{i} \mid \mu+\psi z_{i}, \tau^{2}\right), \quad i=1, \ldots, n \\
z_{i} \stackrel{i n d}{\sim} \mathrm{N}\left(z_{i} \mid 0,1\right) 1_{[0, \infty)}\left(z_{i}\right), \quad i=1, \ldots, n
\end{gathered}
$$

The Bayesian model is completed with (conditionally conjugate) priors for the NS mass distribution parameters. Specifically, we place a bivariate normal prior, $\mathrm{N}\left((\mu, \psi)^{T} \mid \theta, \Sigma\right)$, on $(\mu, \psi)$ with mean vector $\theta$ and covariance matrix $\Sigma$, and an inverse-gamma prior, $\operatorname{IG}\left(\tau^{2} \mid a, b\right)$, on $\tau^{2}$ with shape parameter $a>1$ and scale parameter $b$, such that the prior mean of $\tau^{2}$ is $b /(a-1)$.

\section{A.2. $M C M C$ Posterior Simulation Method}

In addition to the NS mass distribution parameters $\left(\mu, \tau^{2}, \psi\right)$, the model parameters include the individual NS masses $\mathcal{M}_{1}, \ldots, \mathcal{M}_{n}$, as well as the auxiliary random variables $z_{1}, \ldots, z_{n}$. The likelihood function and the priors for $(\mu, \psi)$ and $\tau^{2}$ combine to give the posterior distribution for all model parameters which is proportional to:

$$
\mathrm{N}\left((\mu, \psi)^{T} \mid \theta, \Sigma\right) \operatorname{IG}\left(\tau^{2} \mid a, b\right) \prod_{i=1}^{n}\left\{\operatorname{AN}\left(m_{i}-\mathcal{M}_{i} \mid c_{i}, d_{i}\right) \mathrm{N}\left(\mathcal{M}_{i} \mid \mu+\psi z_{i}, \tau^{2}\right) \mathrm{N}\left(z_{i} \mid 0,1\right) 1_{[0, \infty)}\left(z_{i}\right)\right\} .
$$

We utilize an MCMC posterior simulation algorithm to sample from the posterior distribution. The MCMC algorithm dynamically updates the model parameters by simulating from their full conditional distributions in turn, and upon reaching convergence, the resulting samples are samples from the posterior distribution which may be used for inference. The key benefit of the augmented hierarchical model that includes the auxiliary variables $z_{1}, \ldots, z_{n}$ is that it allows for the use of a Gibbs sampler, which is both efficient and straightforward to implement, since it involves sampling from standard distributions as described below.

For each $i=1, \ldots, n$, the full posterior conditional distribution for $\mathcal{M}_{i}$ is proportional to $\operatorname{AN}\left(m_{i}-\mathcal{M}_{i} \mid c_{i}, d_{i}\right) \mathrm{N}\left(\mathcal{M}_{i} \mid\right.$ $\left.\mu+\psi z_{i}, \tau^{2}\right)$, which can be shown to result in a mixture of two truncated normals. The first truncated normal has mean $\left\{\left(\mu+\psi z_{i}\right) c_{i}^{2} d_{i}^{2}+m_{i} \tau^{2}\right\} /\left(\tau^{2}+c_{i}^{2} d_{i}^{2}\right)$ and variance $\left(\tau^{2} c_{i}^{2} d_{i}^{2}\right) /\left(\tau^{2}+c_{i}^{2} d_{i}^{2}\right)$, and is restricted to the interval $\left(-\infty, m_{i}\right]$. The second truncated normal has mean $\left\{\left(\mu+\psi z_{i}\right)\left(d_{i}^{2} / c_{i}^{2}\right)+m_{i} \tau^{2}\right\} /\left(\tau^{2}+\left(d_{i}^{2} / c_{i}^{2}\right)\right)$ and variance $\left(\tau^{2} d_{i}^{2} / c_{i}^{2}\right) /\left(\tau^{2}+\left(d_{i}^{2} / c_{i}^{2}\right)\right)$, and is restricted to the interval $\left(m_{i}, \infty\right)$. The (unnormalized) weight of the first truncated normal is

$$
\exp \left\{-\frac{\left(\mu+\psi z_{i}-m_{i}\right)^{2}}{2\left(\tau^{2}+c_{i}^{2} d_{i}^{2}\right)}\right\} \cdot \frac{\tau d_{i} c_{i}}{\left(\tau^{2}+d_{i}^{2} c_{i}^{2}\right)^{1 / 2}}\left\{\Phi\left(\frac{m_{i}-\left(\left(\mu+\psi z_{i}\right) c_{i}^{2} d_{i}^{2}+m_{i} \tau^{2}\right) /\left(\tau^{2}+d_{i}^{2} c_{i}^{2}\right)}{\tau d_{i} c_{i} /\left(\tau^{2}+d_{i}^{2} c_{i}^{2}\right)^{1 / 2}}\right)\right\}
$$

and that of the second is

$$
\exp \left\{-\frac{\left(\mu+\psi z_{i}-m_{i}\right)^{2}}{2\left(\tau^{2}+\left(d_{i}^{2} / c_{i}^{2}\right)\right)}\right\} \cdot \frac{\tau d_{i} / c_{i}}{\left(\tau^{2}+\left(d_{i}^{2} / c_{i}^{2}\right)\right)^{1 / 2}}\left\{1-\Phi\left(\frac{m_{i}-\left(\left(\mu+\psi z_{i}\right)\left(d_{i}^{2} / c_{i}^{2}\right)+m_{i} \tau^{2}\right) /\left(\tau^{2}+\left(d_{i}^{2} / c_{i}^{2}\right)\right)}{\left(\tau d_{i} / c_{i}\right) /\left(\tau^{2}+\left(d_{i}^{2} / c_{i}^{2}\right)\right)^{1 / 2}}\right)\right\} .
$$


Each $\mathcal{M}_{i}$ is therefore updated at each MCMC iteration by drawing from a mixture of two truncated normals, with parameters given above.

Next, the full conditional distribution for $z_{i}$ is proportional to $\mathrm{N}\left(\mathcal{M}_{i} \mid \mu+\psi z_{i}, \tau^{2}\right) N\left(z_{i} \mid 0,1\right) 1_{[0, \infty)}\left(z_{i}\right)$, which results in a truncated normal distribution with mean $\left(\mathcal{M}_{i}-\mu\right) \psi /\left(\psi^{2}+\tau^{2}\right)$ and variance $\tau^{2} /\left(\psi^{2}+\tau^{2}\right)$, restricted to $[0, \infty)$.

The NS mass distribution parameters can be sampled with standard updates. The posterior full conditional distribution for $(\mu, \psi)$ can be derived as a bivariate normal distribution,

$$
\mathrm{N}\left((\mu, \psi)^{T} \mid\left(\Sigma^{-1}+Z^{T} Z / \tau^{2}\right)^{-1}\left(\Sigma^{-1} \theta+Z^{T} \mathcal{M} / \tau^{2}\right),\left(\Sigma^{-1}+Z^{T} Z / \tau^{2}\right)^{-1}\right),
$$

where $\mathcal{M}=\left(\mathcal{M}_{1}, \ldots, \mathcal{M}_{n}\right)^{T}$, and $Z$ is an $n \times 2$ matrix with $Z_{i 1}=1$ and $Z_{i 2}=z_{i}$, for $i=1, \ldots, n$. Lastly, the full conditional for $\tau^{2}$ is given by an inverse-gamma distribution with updated shape and scale parameters, $\mathrm{IG}(a+n / 2, b+$ $\left.\sum_{i=1}^{n}\left(\mathcal{M}_{i}-\mu-\psi z_{i}\right)^{2} / 2\right)$.

The Gibbs sampler is therefore implemented with the following general procedure:

- Begin with initial values $\left(\left\{\mathcal{M}_{1}^{(0)}, \ldots, \mathcal{M}_{n}^{(0)}\right\},\left\{z_{1}^{(0)}, \ldots, z_{n}^{(0)}\right\}, \mu^{(0)}, \psi^{(0)}, \tau^{2(0)}\right)$.

- If the current sample at iteration $t$ is $\left(\left\{\mathcal{M}_{1}^{(t)}, \ldots, \mathcal{M}_{n}^{(t)}\right\},\left\{z_{1}^{(t)}, \ldots, z_{n}^{(t)}\right\}, \mu^{(t)}, \psi^{(t)}, \tau^{2(t)}\right)$, obtain the next sample by simulating from the following distributions:

- draw $\mathcal{M}_{i}^{(t+1)}, i=1, \ldots, n$, from a mixture of truncated normal distributions as described above, using the current values of parameters $\mu^{(t)}, \psi^{(t)},\left\{z_{1}^{(t)}, \ldots, z_{n}^{(t)}\right\}$ and $\tau^{2(t)}$

- draw $z_{i}^{(t+1)}, i=1, \ldots, n$, from a normal distribution with mean $\left(\mathcal{M}_{i}^{(t+1)}-\mu^{(t)}\right) \psi^{(t)} /\left(\psi^{2(t)}+\tau^{2(t)}\right)$ and variance $\tau^{2(t)} /\left(\psi^{2(t)}+\tau^{2(t)}\right)$, restricted to the interval $[0, \infty)$

- draw $(\mu, \psi)^{(t+1)}$ jointly from the bivariate normal distribution given in (A3), using the current values of all other parameters

- draw $\tau^{2(t+1)}$ from $\operatorname{IG}\left(a+n / 2, b+\sum_{i=1}^{n}\left(\mathcal{M}_{i}^{(t+1)}-\mu^{(t+1)}-\psi^{(t+1)} z_{i}^{(t+1)}\right)^{2} / 2\right)$

- Repeat the previous step, for $t=1, \ldots, T$.

The chain appeared to converge very rapidly; when two chains were run with different initial values, they met within just a few iterations. There was some autocorrelation present for $\mu$ and $\psi$, but it fell below 0.2 after approximately a lag of 5 , and samples were approximately uncorrelated by a lag of 10 . In light of these results, all inferences are based on 10,000 posterior samples, which came from a longer chain which was thinned, retaining every 10 posterior samples.

\section{A.3. Prior Specification}

Hyperparameters $\theta=\left(\theta_{\mu}, \theta_{\psi}\right)^{T}, \Sigma, a$, and $b$ must be specified in this Bayesian model. First, note that $E(\mathcal{M})=$ $\theta_{\mu}+\theta_{\psi} E(z)$, and the skewness parameter of the NS mass distribution is $\alpha=\psi / \tau$. To avoid favoring skewness in the prior distribution, we set $E(\psi)=\theta_{\psi}=0$, and then set $\theta_{\mu}$ to a reasonable prior location for the NS mass distribution for each system. The set of priors used for the results in $\S 4.2$ had $\theta_{\mu}=1.3$ for the DNS system, and $\theta_{\mu}=1.45$ for the NS-WD system. With $\theta_{\psi}=0$, we have that $\operatorname{Var}(\mathcal{M})=b /(a-1)+\Sigma_{\mu}+\Sigma_{\psi}$, where $\left(\Sigma_{\mu}, \Sigma_{\psi}\right)$ are the elements of matrix $\Sigma$, which is taken to be diagonal. We fix $a=3$ in each set of priors, and for the DNS system we set $b /(a-1)$, $\Sigma_{\mu}$, and $\Sigma_{\psi}$ all to $0.25^{2} / 3$. For the NS-WD system, we set all three expressions to $0.3^{2} / 3$. These result in a fairly dispersed (thus, non-informative) prior distribution for NS masses as demonstrated by the prior interval estimates in Figure 3.

\section{B. POSTERIOR PREDICTIVE MODEL CHECKING RESULTS}

As discussed briefly in $\S 4.3$, for model checking we work with the posterior predictive distributions for replicated responses for each pulsar. For each $i=1, \ldots, n$, the corresponding expression is given by

$$
\mathcal{P}\left(m_{i}^{r e p} \mid \text { data }\right)=\int \operatorname{AN}\left(m_{i}^{r e p}-\mathcal{M}_{i} \mid c_{i}, d_{i}\right) p\left(\mathcal{M}_{i} \mid \text { data }\right) \mathrm{d} \mathcal{M}_{i} .
$$

This distribution can be sampled by drawing a random variate from the asymmetric normal distribution in the integral representation above for each posterior sample of $\mathcal{M}_{i}$. Figure 4 provides histograms of these posterior predictive samples for replicate responses associated with each pulsar from the DNS and NS-WD systems. Again, the results suggest good predictive performance for the model. 


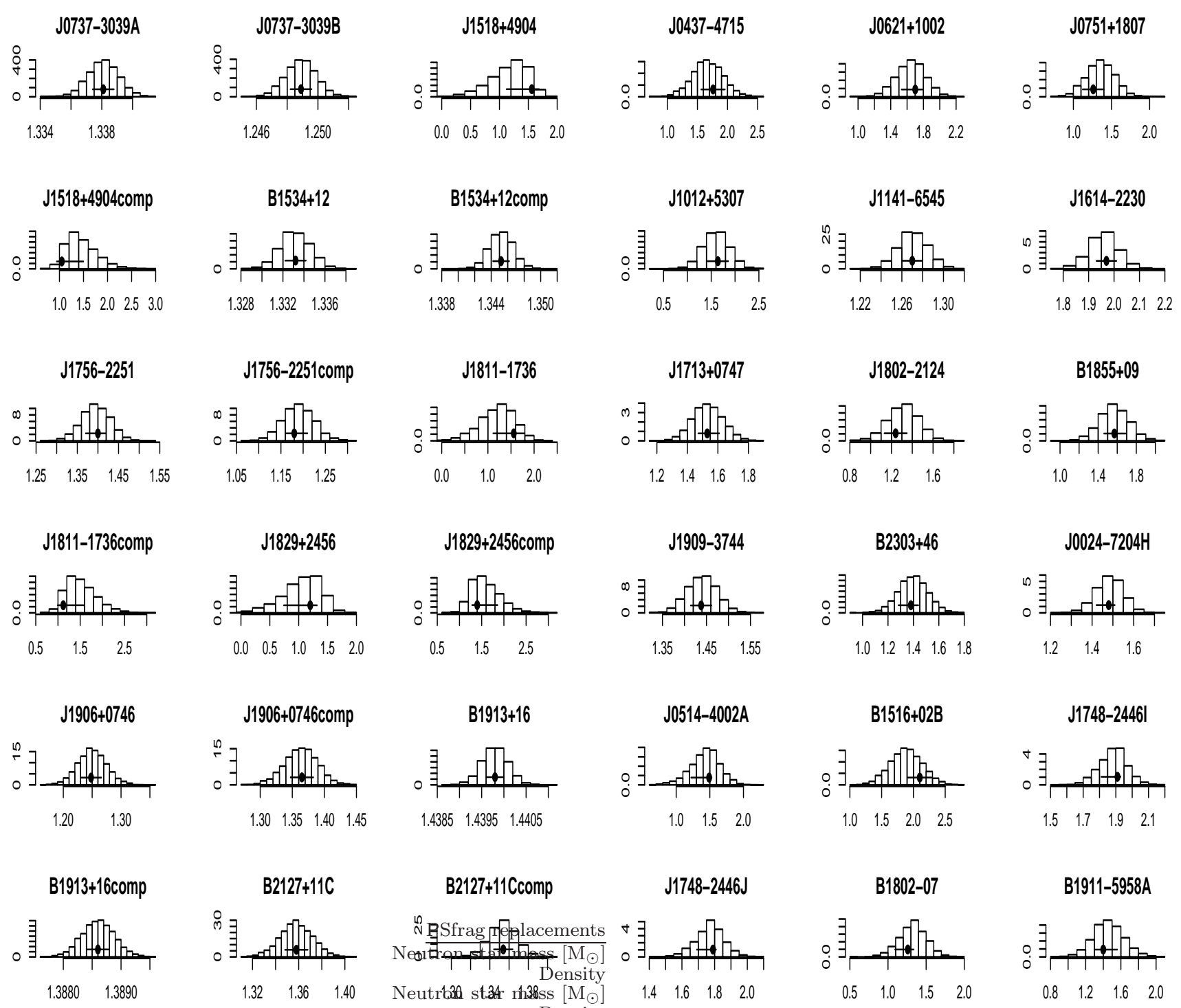

Figure 4. Histograms of posterior predictive distributions for replicated responses for each pulsar. Columns 1-3 plot results for each observed pulsar mass estimate $m_{i}$ in DNS systems, and columns 4-6 for the NS-WD systems. The corresponding data for the measured NS masses, including both the estimates and error bars, are displayed in each panel. 